\title{
Perceived Brand Luckiness: Scale Development and Validation: An Abstract
}

\author{
Jiani Jiang and Miao Zhao
}

\begin{abstract}
Luck-related concepts such as luck, belief in luck, beliefs about luck, personal luck, and luck beliefs along with their impacts have been widely researched by psychologists and personality researchers (Langer 1975; Presson and Benassi 1996; Andre 2006; Bridgstock et al. 2011; Maltby et al. 2008; Young et al. 2009; Thompson and Predergast 2013; Darke and Freedman 1997; Wohl and Enzle 2002; Wohl et al. 2011). However, individual's luck-related beliefs and their impacts on consumer preferences and decision-making have received little attention. The lack of investigation regarding luck-related beliefs is more surprising given their significant influences in the discipline (Kramer and Block 2008). Consumers interact with thousands of products and brands in their lives and tend to associate products and brands with the outcomes of certain events on a day-to-day basis (Hamerman and Johar 2013).

The authors argue that consumers may perceive certain brands luckier than the others and believe that buying/consuming the lucky brands will lead to positive outcomes; therefore, they are more likely to be loyal to and repurchase the lucky brands. Unfortunately, no study has conceptualized and empirically tested consumers' perceived luckiness of a specific brand. Consequently, it has been difficult for both researchers and practitioners to understand whether belief in luck can be transferred to a brand and to further predict their commitment to the brand (e.g., brand loyalty) and their willingness to make financial expenses in order to obtain it (e.g., purchase intention). As a result, a clear definition and reliable measurement to access consumers' perception of luckiness associated with a brand is needed.

Therefore, the present research conceptualizes, develops, and validates a unidimensional eight-item scale for measuring perceived brand luckiness. Subsequent empirical results show belief in luck relates positively to perceived brand luckiness, which in turn relates positively to purchase intention and brand loyalty.
\end{abstract}

\author{
J. Jiang $(\bowtie)$ \\ New Mexico State University, Las Cruces, NM, USA \\ e-mail: jjiang@nmsu.edu
}

M. Zhao

Roger Williams University, Bristol, RI, USA

e-mail: mzhao@rwu.edu 